\title{
Variación del crecimiento en vivero entre procedencias de Pinus pseudostrobus Lindl.
}

\section{Growth variation in nursery among provenances of Pinus pseudostrobus Lindl.}

\author{
Daniel E. Villegas-Jiménez ${ }^{1}$, Gerardo Rodríguez-Ortiz ${ }^{1}$, José L. Chávez-Serviaª , José R. Enríquez- \\ Del-Valle ${ }^{1} \&$ José C. Carrillo-Rodriguez ${ }^{1}$
}

${ }^{1}$ Instituto Tecnológico del Valle de Oaxaca, Ex-Hacienda de Nazareno, Santa Cruz Xoxocotlán, Oaxaca, México, C.P. 71230. ${ }^{2}$ Instituto Politécnico Nacional, CIIDIR Unidad Oaxaca, Hornos \# 1003 Santa Cruz Xoxocotlan, Oaxaca, México. C.P. 71230. *jchavezs@ipn.mx

\begin{abstract}
RESUMEN
Pinus pseudotrobus es una especie con aprovechamiento maderable, distribuida en un amplio gradiente altitudinal, de 1.600 a $3.250 \mathrm{~m}$, lo que sugiere una varianza genética que puede ser aprovechada mediante la selección de árboles que provean semillas que generen plantas con altas tasas de crecimiento relativo. Por lo anterior, se evaluó la sobrevivencia y crecimiento inicial de Pinus pseudostrobus en vivero, con base en la variación de progenies de 38 árboles sobresalientes (procedencias) en rodales naturales de la Sierra Norte de Oaxaca, México. La siembra se realizó en febrero de 2013 en un vivero localizado en Ixtlán de Juárez, cercano a la región de colecta, a $2030 \mathrm{msnm}$ y un clima templado. Además de evaluar la sobrevivencia de 54 a 166 días después de la siembra (dds), se estimaron cinco lecturas de altura de planta, diámetro basal de tallo y cuatro lecturas de número de braquiblastos, y se calculó la tasa de crecimiento relativo para altura de planta. Mediante un análisis de varianza y comparación de medias se determinaron diferencias significativas entre procedencias, en todas las variables. En la evaluación se manifestó un crecimiento exponencial de plantas en vivero, con un promedio de 0,8 a $1,5 \mathrm{~mm}$ de altura/día de 54 a 166 dds y un promedio de 1 a 2 braquiblastos en ramillas primarias y secundarias por día en un intervalo de 82 a 166 dds. Del total de procedencias, cuatro de ellas mostraron una alta tasa de crecimiento.
\end{abstract}

Palabras clave: Bosque templado, tasa de crecimiento relativo, variación fenotípica.

\begin{abstract}
Pinus pseudostrobus is a species with timber harvesting, distributed over a wide altitudinal gradient of 1,600-3,250 m, suggesting a genetic variance, which can be exploited by selecting trees that provide seeds in order to produce plants with high relative growth rates. Therefore, survival and early growth of Pinus pseudostrobus were evaluated under nursery conditions, based on the variation in offsprings of 38 outstanding trees (provenances) in natural stands from Sierra Norte of Oaxaca, Mexico. Seeds were sowing in February 2013 at a nursery located in Ixtlan de Juarez, near the collection site, to 2030 masl and a temperate climate. Survival from 54-166 days after planting (dap) was evaluated, five readings of plant height, basal stem diameter, four readings of number of brachyblasts were recorded, and the relative growth rate for plant height was estimated. Using analysis of variance and mean tests, significant differences among provenances were determined in all variables. In the evaluation, the plants in the nursery showed an exponential growth, an average of 0,8 to $1,5 \mathrm{~mm}$ in height/day of 54-166 dap and an average of 1-2 brachyblasts on primary and secondary branches per day in a range of 82-166 dap. From total provenances, four showed high growing rate.
\end{abstract}

KEYwORDS: Template forest, relative growth rate, phenotypic variation.

\section{INTRODUCCIÓN}

En México los bosques y selvas ocupan del 29 al 32,7\% de la superficie nacional (1.941 mill. de $\mathrm{km}^{2}$ ) con una proporción de bosque/población humana de 0,3 a 0,8 ha por individuo, no obstante debido a tasas de deforestación de
370.000 hasta 1.500 .000 ha/año $(0,7$ a $2 \%$ anual $)$, existe una pérdida de biodiversidad, de valor del bosque maderable y de sus funciones ecológicas (Toledo 1989, Palacio-Prieto et al. 2000, Velázquez et al. 2002, Díaz-Gallegos et al. 2008). A pesar de diversos esfuerzos por reforestar, las plantaciones con fines de explotación no superan el 1\% del 
total (Velázquez et al. 2002, Sáenz-Romero et al. 2003). Esto indica que se deben incrementar los esfuerzos para realizar plantaciones con fines de explotación forestal, entre otras medidas, y con ello disminuir la tasa de deforestación.

El aprovechamiento forestal en México se basa esencialmente en la explotación de los bosques naturales de gran diversidad de Pinus y Quercus. En el género Pinus se agrupan 47 especies, 3 subespecies y 22 variedades botánicas; 26 de estas especies son endémicas de México y se distribuyen en cinco regiones denominadas como: Baja California Norte y Sur, Sierra Madre Occidental, Sierra Madre Oriental, Faja Volcánica (zona central de México) y Sierra Madre del Sur donde se incluyen los estados de Guerrero, Oaxaca, Chiapas, Campeche y Quintana Roo con altitudes desde el nivel del mar (Pinus caribaea Morelet var. hondurensis (Sénécl.) W.H.G.) a regiones de alta montaña (Pinus hartwegii Lindl.) de 4.300 m s.n.m. (Sáenz-Romero et al. 2003, Sánchez-González 2008, Delgado et al. 2013).

Pinus pseudostrobus Lindl. es una especie endémica de México con alta variación genética altitudinal, se explota como madera en los rodales naturales, y en algunos sitios se encuentra asociada con P. montezume Lamb., razón por la que se han evidenciado cruzamientos interespecíficos, permitiendo el incremento de la variación genética como producto del flujo genético entre especies (Stead 1983, Delgado \& Piñeiro 2002, Viveros-Viveros et al. 2005a, Delgado et al. 2007, Sáenz-Romero et al. 2012, CastellanosAcuña et al. 2013). P. pseudostrobus es una especie con potencial para reforestación, agroforestería y plantaciones comerciales maderables por la calidad de su madera y rápido crecimiento, aunado a la alta diversidad genética y amplia distribución altitudinal de 1.600 a $3.250 \mathrm{msnm}$; prospera en climas templados a templados cálidos con temperaturas que oscilan de -9.0 a $40{ }^{\circ} \mathrm{C}$, y en México se distribuye entre $17^{\circ} 15^{\prime}$ a $29^{\circ} 25^{\prime} \mathrm{N}$ y $92^{\circ} 05^{\prime}$ a $108^{\circ} 35^{\prime}$ O (Equiluz 1982 , Sáenz et al. 2011, Cambrón et al. 2013). La presencia de alta diversidad genética y amplia distribución, son elementos que ayudan a sustentar un programa de mejoramiento genético de $P$. pseudostrobus a largo plazo pero se requiere incrementar el conocimiento sobre biología de la especie, dinámicas poblacionales y caracteres heredables con fines de explotación maderable.

Estudios previos de P. pseudostrobus han documentado que posee cierta resistencia a plagas como Heterotermes sp. (Reyes et al. 1995), alta variación en porcentajes de germinación de semillas con diferentes procedencias (Ramírez-García et al. 2001), alto crecimiento inicial en condiciones de competencia en ensayos de campo (Cambrón et al. 2013, Sáenz et al. 2011); en rodales naturales puede alcanzar alturas entre 10 y $50 \mathrm{~m}$ a una edad de 18 a 40 años (Sáenz et al. 2011). Estás y otras características son relevantes para la selección de progenitores sobresalientes para emprender un programa de mejoramiento genético de la especie.
La selección de progenitores sobresalientes de alto valor genético de P. pseudostrobus, con fines de mejoramiento, tiene como base la recolección de semilla de árboles promisorios en rodales naturales, germinación y evaluación temprana del crecimiento en vivero, evaluación de tasa de crecimiento y producción de biomasa en los primeros años después del trasplante en campo y la evaluación dasométrica en estado adulto de la progenie en función de las procedencias (Ramírez-García et al. 2001, HernándezCarmona et al. 2003, Villar et al. 2004, Viveros-Viveros et al. 2005a, Viveros-Viveros et al. 2006, Sáenz et al. 2011, Muñoz et al. 2011). Debido a largos periodos que implica el mejoramiento genético forestal, es evidente que se busca determinar los caracteres genéticos de alta heredabilidad para hacer eficiente la selección temprana de progenitores (Farfán et al. 2002). En este contexto, se evaluó la sobrevivencia y crecimiento inicial de Pinus pseudostrobus en vivero, con base en la variación de progenies de 38 árboles considerados sobresalientes (procedencias) en un rodal natural de la Sierra Norte de Oaxaca, México.

\section{MATERIALES Y MÉTODOS}

\section{Material VEgetal Y EVALUACión DE CRECIMIENTO}

En diferentes recorridos por los bosques de pino-encino, entre altitudes de 2.440 y 2.960 m s.n.m. de la Sierra Norte de Oaxaca, México, de octubre de 2012 a enero de 2013, se marcaron y obtuvo semilla de 38 árboles de $P$. pseudostrobus, las que se designaron como procedencias de progenitores sobresalientes con base en su volumen comercial maderable, mejores características dasométricas y de ubicación geográfica (Tabla I y Fig. 1). La edad de cada individuo se determinó contando los anillos de crecimiento en virutas obtenidas con el taladro de Pressler (Haglof, serie BS102B); el diámetro normal fue medido con clinómetro digital (Haglof, modelo EC II); el diámetro de copa con longímetro de $30 \mathrm{~m}$; y los datos de localización con geoposicionador satelital (Garmin, ETrex 30). La rectitud del fuste se simuló de forma visual a una línea recta y el volumen de madera fue estimado mediante la ecuación (TIASA, 1993):,$V=0.00004791 \times D N^{1.62263717} \times A T^{1.28112040}$ donde: $\mathrm{V}=$ volumen $\left(\mathrm{m}^{3}\right), \mathrm{DN}=$ diámetro normal $(\mathrm{cm}), \mathrm{AT}$ $=$ altura total $(\mathrm{m})$.

Las semillas de cada progenitor (=procedencias) fueron sembradas en el mes de febrero de 2013 en charolas de plástico de 49 cavidades de $175 \mathrm{~cm}^{3}$ que contenían un sustrato compuesto por mezcla de turba $(40 \%)$, vermiculita $(30 \%)$ y perlita $(30 \%)$, en un vivero forestal de malla sombra en Ixtlán de Juárez en la Sierra Norte de Oaxaca (17 $20^{\circ}$, LN y $96^{\circ} 29^{\prime}$ LO, a 2.030 m s.n.m.). Las características climatológicas de la ubicación del vivero son $18,3{ }^{\circ} \mathrm{C}$ y $759.3 \mathrm{~mm}$ de temperatura y precipitación medias anuales, 
respectivamente (Zacarías-Eslava y del Castillo 2010). La distribución de procedencias se hizo en charola por árbol (=unidad experimental) en un arreglo completamente al azar con tres repeticiones.

En cada unidad experimental se evaluó el porcentaje de emergencia a 54 días después de la siembra (dds) y porcentaje de sobrevivencia a 166 dds. También se tomó una muestra aleatoria de cinco plántulas por unidad experimental evitando las plántulas de orilla como "efecto de borde", la altura se midió $(\mathrm{cm})$ desde la base del tallo hasta la yema apical con una regla con aproximación a milímetros, el diámetro basal de tallo $(\mathrm{mm})$ se midió en la base del tallo con un vernier Scala 222A® (Gimbel Mexicana S.A. de C.V., México, D. F) con aproximación a milímetros a 54, 82, 110, 138 y 166 dds. Posteriormente, con los valores de altura de planta se estimó la tasa de crecimiento relativo (TCR) con base en la siguiente expresión (Villar et al. 2004):TCR=[ln (altura 2)- $\ln ($ altura 1$)] /[$ Tiempo 2-Tiempo 1]; donde: $\mathrm{TCR}=$ tasa de crecimiento relativo $\left(\mathrm{cm} \mathrm{día}^{-1}\right), \ln ($ altura 2$)=$ altura de planta a $166 \mathrm{dds}(\mathrm{cm})($ tiempo 2$), \ln ($ altura 1$)=$ altura de planta a 54 dds (tiempo 1). Complementariamente, se evaluó el número de braquiblastos de acículas sobre ramillas primarias y secundarias a $82,110,138$ y $166 \mathrm{dds}$, únicamente a dos plántulas por procedencia, seleccionadas aleatoriamente debido a la dificultad del conteo de braquiblastos. Las fechas de medición (dds) fueron programadas de manera mensual, iniciando aproximadamente en el tercer mes de crecimiento, una vez que se mantuvo definido el número de plántulas emergidas y en crecimiento activo; y culminando poco antes que las plántulas se llevaran al sitio de plantación. Por experiencias previas, antes de esa fase inicial de crecimiento se observaban muertes de plántulas, razón por la que la primera lectura se realizó 54 dds.

\section{AnÁlisis ESTADÍSTICO}

Se integró una base de datos por procedencia, repetición y plantas cuando fue el caso. A los valores de porcentaje de emergencia y supervivencia, se aplicó la transformación arcoseno raíz $(\% / 100)$ y para el conteo de braquiblastos se aplicó la transformación raíz(x); después, se realizaron los análisis de varianza mediante el modelo lineal del diseño abajo descrito para evaluar las diferencias entre procedencias de $P$. pseudostrobus a 54 y $166 \mathrm{dds}$, y posteriormente comparaciones de medias por el método de Tukey $(\mathrm{P}<0.05)$. Los análisis estadísticos fueron desarrollados en el paquete estadístico $\mathrm{r}($. El modelo lineal utilizado fue el siguiente (Sahagún, 1998): $Y_{i j k}=\mu+P_{\mathrm{i}}+A_{k}\left(P_{i}\right)+\varepsilon_{i j k}$; donde: $Y_{i j k}=$ variables respuesta (en i-ésima procedencia, j-ésima repetición y k-ésimo árbol), $\mu=$ media general, $P_{i}=$ efecto de procedencias, $A_{k}\left(P_{i}\right)=$ plantas anidadas en procedencias (a excepción de emergencia y sobrevivencia), y $\varepsilon_{i j k}=$ error experimental asociado a las variables descritas. Complementariamente, se hizo un análisis gráfico descriptivo de los patrones de crecimiento donde se contrastaron las procedencias con mayores y menores tasas de crecimiento en altura de planta y número de ramas primarias y secundarias desde 54 a 166 dds.

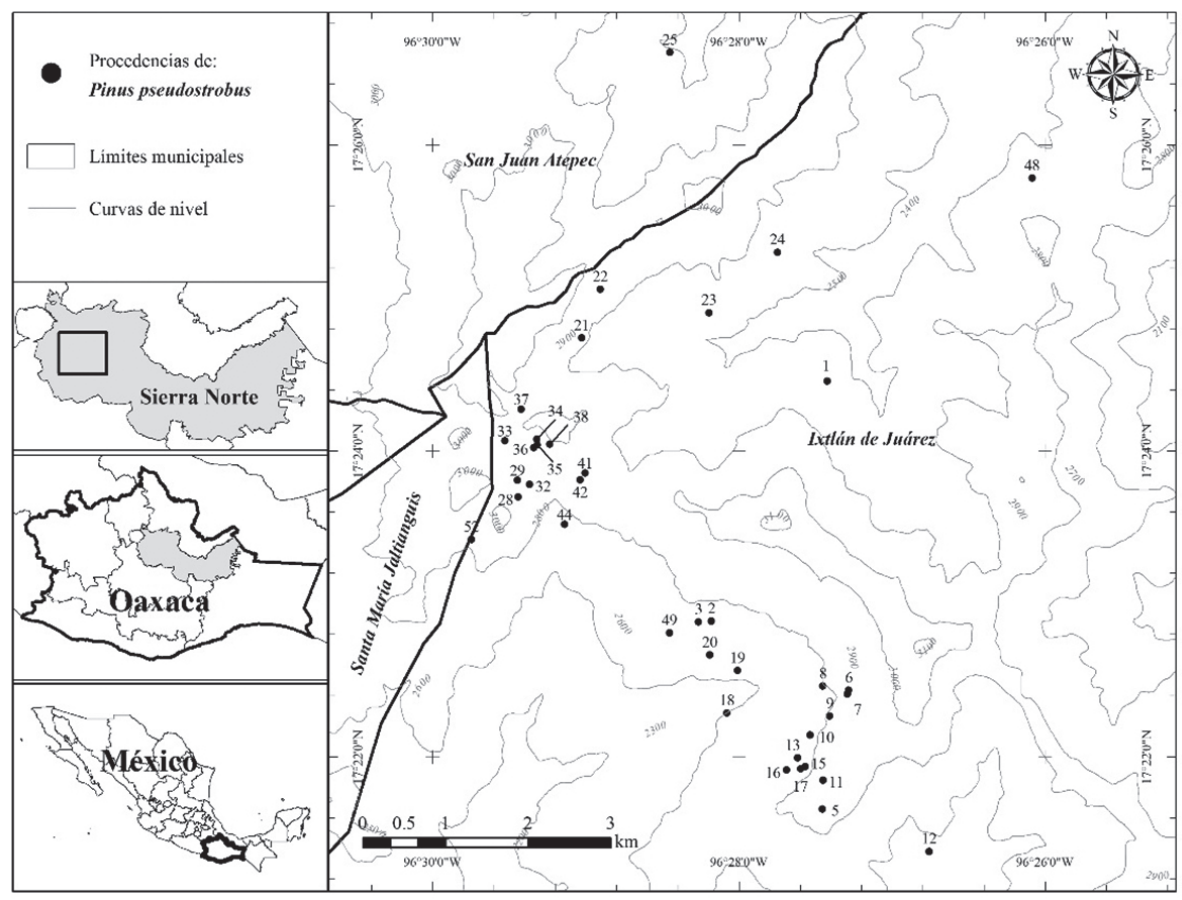

Figura 1. Localización de las procedencias de Pinus pseudostrobus en la Sierra Norte de Oaxaca, México.

Figure 1. Location of the provenances of Pinus pseudostrobus in the Sierra Norte de Oaxaca, Mexico. 
Gayana Bot. 73(1), 2016

TABLA I. Características de progenitores/procedencias de Pinus pseudostrobus de Ixtlán, Oaxaca, México.

TABLE I. Characteristics of parents/provenances of Pinus pseudostrobus of Ixtlan, Oaxaca, México.

\begin{tabular}{|c|c|c|c|c|c|c|c|c|}
\hline PROGENITOR $^{\S}$ & $\begin{array}{l}\text { EDAD } \\
\text { (años) }\end{array}$ & $\begin{array}{c}\text { DIÁMETRO } \\
\text { NORMAL } \\
(\mathrm{cm})\end{array}$ & $\begin{array}{l}\text { RECTITUD DEL } \\
\text { FUSTE }\end{array}$ & $\begin{array}{c}\text { DiÁMETRO DE } \\
\text { COPA }(\mathrm{m})\end{array}$ & $\begin{array}{c}\text { VOLUMEN } \\
\text { ESTIMADO }\left(\mathrm{m}^{3}\right)\end{array}$ & $\begin{array}{l}\text { Altitud } \\
\text { DEL SITIO } \\
\quad(\mathrm{m})\end{array}$ & LATITUD NORTE & $\begin{array}{l}\text { LONGITUD } \\
\text { OESTE }\end{array}$ \\
\hline 1 & 60 & 84,0 & 41 & 4,0 & 8,2146 & 2540 & $17^{\circ} 24^{\prime} 27.4^{\prime \prime}$ & $96^{\circ} 27^{\prime} 25.3^{\prime \prime}$ \\
\hline 2 & 33 & 61,5 & 33 & 4,0 & 3,7349 & 2600 & $17^{\circ} 22^{\prime} 53.3^{\prime \prime}$ & $96^{\circ} 28^{\prime} 10.6^{\prime \prime}$ \\
\hline 3 & 72 & 83,0 & 41 & 4,0 & 8,0333 & 2640 & $17^{\circ} 22^{\prime} 53.0^{\prime \prime}$ & $96^{\circ} 28^{\prime} 15.7^{\prime \prime}$ \\
\hline 5 & 50 & 60,0 & 38 & 4,0 & 4,3385 & 2590 & $17^{\circ} 21^{\prime} 39.6^{\prime \prime}$ & $96^{\circ} 27^{\prime} 27.2^{\prime \prime}$ \\
\hline 6 & 82 & 53,0 & 36 & 4,3 & 3,0761 & 2780 & $17^{\circ} 22^{\prime} 26.2^{\prime \prime}$ & $96^{\circ} 27^{\prime} 16.9^{\prime \prime}$ \\
\hline 7 & 85 & 75,0 & 37 & 4,0 & 6,0299 & 2780 & $17^{\circ} 22^{\prime} 24.7^{\prime \prime}$ & $96^{\circ} 27^{\prime} 17.3^{\prime \prime}$ \\
\hline 8 & 54 & 60,0 & 34 & 4,0 & 3,6701 & 2710 & $17^{\circ} 22^{\prime} 27.8^{\prime \prime}$ & $96^{\circ} 27^{\prime} 27.0^{\prime \prime}$ \\
\hline 9 & 80 & 69,0 & 44 & 3,5 & 6,0911 & 2700 & $17^{\circ} 22^{\prime} 16.0^{\prime \prime}$ & $96^{\circ} 27^{\prime} 24.3^{\prime \prime}$ \\
\hline 10 & 70 & 80,0 & 35 & 4,0 & 6,4489 & 2670 & $17^{\circ} 22^{\prime} 08.6^{\prime \prime}$ & $96^{\circ} 27^{\prime} 32.0^{\prime \prime}$ \\
\hline 11 & 83 & 72,0 & 45 & 4,0 & 6,7368 & 2680 & $17^{\circ} 21^{\prime} 50.8^{\prime \prime}$ & $96^{\circ} 27^{\prime} 26.8^{\prime \prime}$ \\
\hline 12 & 70 & 85,0 & 43 & 4,0 & 8,7885 & 2640 & $17^{\circ} 21^{\prime} 22.9^{\prime \prime}$ & $96^{\circ} 26^{\prime} 45.3^{\prime \prime}$ \\
\hline 13 & 77 & 79,5 & 38 & 4,0 & 6,8948 & 2690 & $17^{\circ} 21^{\prime} 59.6^{\prime \prime}$ & $96^{\circ} 27^{\prime} 36.8^{\prime \prime}$ \\
\hline 15 & 53 & 70,0 & 38 & 3,5 & 5,4393 & 2680 & $17^{\circ} 21^{\prime} 56.2^{\prime \prime}$ & $96^{\circ} 27^{\prime} 33.8^{\prime \prime}$ \\
\hline 16 & 57 & 81,0 & 37 & 3,5 & 6,9596 & 2660 & $17^{\circ} 21^{\prime} 54.9^{\prime \prime}$ & $96^{\circ} 27^{\prime} 41.2^{\prime \prime}$ \\
\hline 17 & 40 & 65,0 & 28 & 4,0 & 3,5394 & 2670 & $17^{\circ} 21^{\prime} 55.3^{\prime \prime}$ & $96^{\circ} 27^{\prime} 35.6^{\prime \prime}$ \\
\hline 18 & 77 & 76,0 & 41 & 4,0 & 6,8171 & 2440 & $17^{\circ} 22^{\prime} 17.2^{\prime \prime}$ & $96^{\circ} 28^{\prime} 04.6^{\prime \prime}$ \\
\hline 19 & 50 & 48,0 & 32 & 4,3 & 2,2854 & 2490 & $17^{\circ} 22^{\prime} 33.9^{\prime \prime}$ & $96^{\circ} 28^{\prime} 00.4^{\prime \prime}$ \\
\hline 20 & 44 & 57,0 & 30 & 4,0 & 2,9598 & 2530 & $17^{\circ} 22^{\prime} 40.0^{\prime \prime}$ & $96^{\circ} 28^{\prime} 11.3^{\prime \prime}$ \\
\hline 21 & 51 & 64,0 & 33 & 3,8 & 4,0227 & 2780 & $17^{\circ} 24^{\prime} 44.4^{\prime \prime}$ & $96^{\circ} 29^{\prime} 01.4$ " \\
\hline 22 & 69 & 58,0 & 42 & 4,0 & 4,2158 & 2830 & $17^{\circ} 25^{\prime} 03.5^{\prime \prime}$ & $96^{\circ} 28^{\prime} 54.2^{\prime \prime}$ \\
\hline 23 & 44 & 52,5 & 39 & 4,0 & 3,2623 & 2560 & $17^{\circ} 24^{\prime} 54.3^{\prime \prime}$ & $96^{\circ} 28^{\prime} 11.6^{\prime \prime}$ \\
\hline 24 & 50 & 50,0 & 33 & 4,1 & 2,5396 & 2610 & $17^{\circ} 25^{\prime} 18.0^{\prime \prime}$ & $96^{\circ} 27^{\prime} 44.7^{\prime \prime}$ \\
\hline 25 & 89 & 70,0 & 43 & 4,0 & 6,1208 & 2720 & $17^{\circ} 26^{\prime} 36.4^{\prime \prime}$ & $96^{\circ} 28^{\prime} 26.9^{\prime \prime}$ \\
\hline 28 & 64 & 58,6 & 30 & 4,0 & 3,1165 & 2860 & $17^{\circ} 23^{\prime} 42.0^{\prime \prime}$ & $96^{\circ} 29^{\prime} 26.3^{\prime \prime}$ \\
\hline 29 & 58 & 68,4 & 34 & 4,0 & 4,6849 & 2875 & $17^{\circ} 23^{\prime} 48.6^{\prime \prime}$ & $96^{\circ} 29^{\prime} 26.6^{\prime \prime}$ \\
\hline 32 & 67 & 80,2 & 38 & 4,0 & 7,0083 & 2855 & $17^{\circ} 23^{\prime} 46.9^{\prime \prime}$ & $96^{\circ} 29^{\prime} 21.9^{\prime \prime}$ \\
\hline 33 & 80 & 73,2 & 37 & 4,0 & 5,7630 & 2885 & $17^{\circ} 24^{\prime} 04.0^{\prime \prime}$ & $96^{\circ} 29^{\prime} 31.5^{\prime \prime}$ \\
\hline 34 & 65 & 52,5 & 31 & 3,8 & 2,6201 & 2915 & $17^{\circ} 24^{\prime} 04.6^{\prime \prime}$ & $96^{\circ} 29^{\prime} 19.1$ " \\
\hline 35 & 60 & 62,4 & 38 & 3,7 & 4,3908 & 2895 & $17^{\circ} 24^{\prime} 02.4^{\prime \prime}$ & $96^{\circ} 29^{\prime} 18.9^{\prime \prime}$ \\
\hline 36 & 69 & 69,4 & 42 & 4,1 & 5,8895 & 2895 & $17^{\circ} 24^{\prime} 01.4^{\prime \prime}$ & $96^{\circ} 29^{\prime} 20.2^{\prime \prime}$ \\
\hline 37 & 98 & 95,5 & 43 & 4,0 & 10,919 & 2900 & $17^{\circ} 24^{\prime} 16.4^{\prime \prime}$ & $96^{\circ} 29^{\prime} 25.2^{\prime \prime}$ \\
\hline 38 & 53 & 50,3 & 37 & 4,2 & 2,8645 & 2960 & $17^{\circ} 24^{\prime} 02.7^{\prime \prime}$ & $96^{\circ} 29^{\prime} 13.9^{\prime \prime}$ \\
\hline 41 & 64 & 87,0 & 39 & 4,0 & 8,3607 & 2930 & $17^{\circ} 23^{\prime} 51.4^{\prime \prime}$ & $96^{\circ} 29^{\prime} 00.1^{\prime \prime}$ \\
\hline 42 & 65 & 86,0 & 43 & 3,7 & 8,9822 & 2895 & $17^{\circ} 23^{\prime} 48.7^{\prime \prime}$ & $96^{\circ} 29^{\prime} 02.0^{\prime \prime}$ \\
\hline 44 & 45 & 55,5 & 31 & 4,4 & 2,9059 & 2710 & $17^{\circ} 23^{\prime} 31.2^{\prime \prime}$ & $96^{\circ} 29^{\prime} 08.2^{\prime \prime}$ \\
\hline 48 & 43 & 74,5 & 37 & 4,3 & 5,9552 & 2530 & $17^{\circ} 25^{\prime} 47.1^{\prime \prime}$ & $96^{\circ} 26^{\prime} 04.9$ " \\
\hline 49 & 48 & 62,1 & 29 & 4,0 & 3,3616 & 2620 & $17^{\circ} 22^{\prime} 48.7^{\prime \prime}$ & $96^{\circ} 28^{\prime} 27.1^{\prime \prime}$ \\
\hline 52 & 47 & 60,2 & 39 & 4,5 & 4,2099 & 2760 & $17^{\circ} 23^{\prime} 25.3^{\prime \prime}$ & $96^{\circ} 29^{\prime} 44.7^{\prime \prime}$ \\
\hline
\end{tabular}

§Se respetó la nomenclatura asignada por los Servicios Técnicos en la selección inicial./ It was respected the nomenclature referenced by the Technical Services in the initial selection. 


\section{RESULTADOS}

El crecimiento en vivero de plántulas $P$. pseudostrobus de Ixtlán, Oaxaca, mostraron que las procedencias 0 progenitores presentaron diferencias en la velocidad de crecimiento de altura de planta; las procedencias 17, 9, 28, 2 y 25 se diferenciaron desde 82 dds del grupo de crecimiento más bajo $19,35,37,23$ y 33, y a 166 dds las diferencias fueron de 5 a $10 \mathrm{~cm}$ (Fig. 2). Los resultados muestran que los progenitores representados pueden conferir a la progenie diferencias en crecimiento inicial de plántulas.

Las procedencias de $P$. pseudostrobus también presentaron diferencias en velocidad de emisión de braquiblastos sobre ramillas primarias y ramillas secundarias: 17 y 41 produjeron el mayor número de braquiblastos, contrariamente 11 y 18 generaron el menor número de las mismas (Fig. 3); todo esto entre 82 y 166 días después de la siembra. La producción de mayor cantidad de braquiblastos está asociada con mayor área fotosintéticamente activa, y consecuentemente mayor aprovechamiento de luz.

En los análisis de varianza se detectaron diferencias significativas $(p<0,05)$ entre procedencias para todas las variables evaluadas a 54, 82 y $166 \mathrm{dds}$, excepto en número de braquiblastos en ramillas primarias a 82 dds. Otro aspecto relevante del análisis de varianza fue que no hubo diferencias significativas entre individuos dentro de procedencias (árboles anidados en procedencias) para todas las variables evaluadas, incluyendo la tasa de crecimiento relativo (Tabla II). Los resultados indican que dentro de cada procedencia las plantas presentaron un comportamiento uniforme y ayuda a obtener el efecto promedio de las procedencias. Los valores del coeficiente de variación calculados indican que en número de ramas secundarias es un carácter altamente variable $(\mathrm{CV}>30 \%)$. En este sentido, las diferencias obtenidas en repeticiones se debieron a las condiciones variables en vivero para la producción de ramillas secundarias.

La velocidad de crecimiento inicial de las plantas en vivero está asociada con la capacidad de aprovechar los elementos del medio como son agua, luz y nutrientes. Así, a 54 días de la siembra (dds) las procedencias 13 y 15 presentaron un mayor crecimiento en altura de planta $(4,4$ a $4,5 \mathrm{~cm})$ que las procedencias $6,11,18$ y $35(3,1$ a 3,4 $\mathrm{cm})$. No obstante, el crecimiento mayor a 54 dds no reflejó el mismo patrón a $166 \mathrm{dds}$, ya que la procedencias 1, 2, 9, 17, 28 y 38 sí mostraron el mayor crecimiento $(18,7$ a 20,1

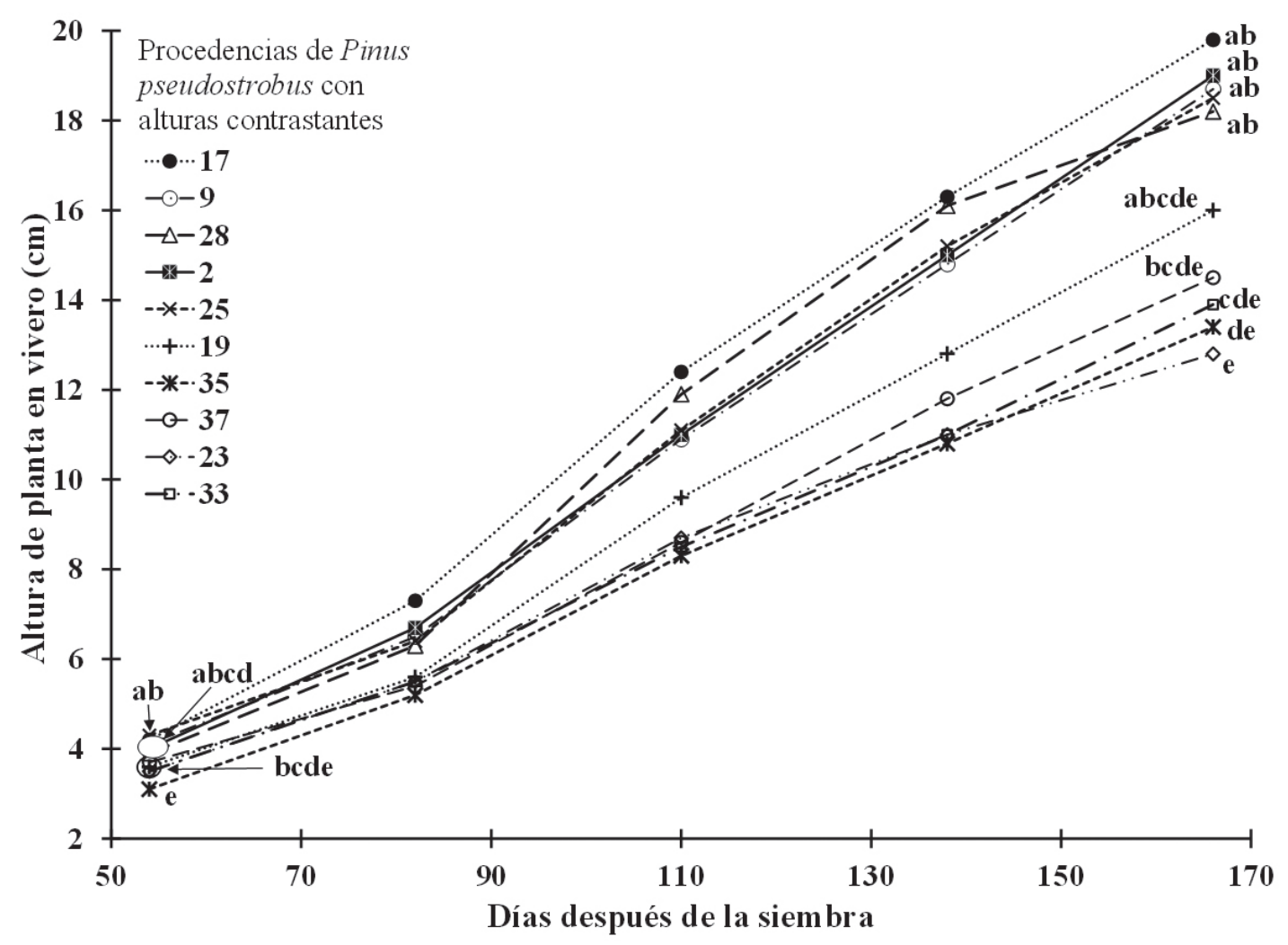

FIGURA 2. Patrones de crecimiento en altura de planta de 10 procedencias/progenitores de Pinus pseudostrobus contrastantes en crecimiento de altura de planta alcanzada, bajo condiciones de vivero. Promedios con letras iguales no difieren estadísticamente (Tukey, P 0.05).

Figure 2. Patterns of growth in plant height of 10 provenances/parents in contrasting Pinus pseudostrobus plant height growth under greenhouse conditions. Averages with similar letter are not statistically different (Tukey, $\mathrm{P}<0.05$ ). 


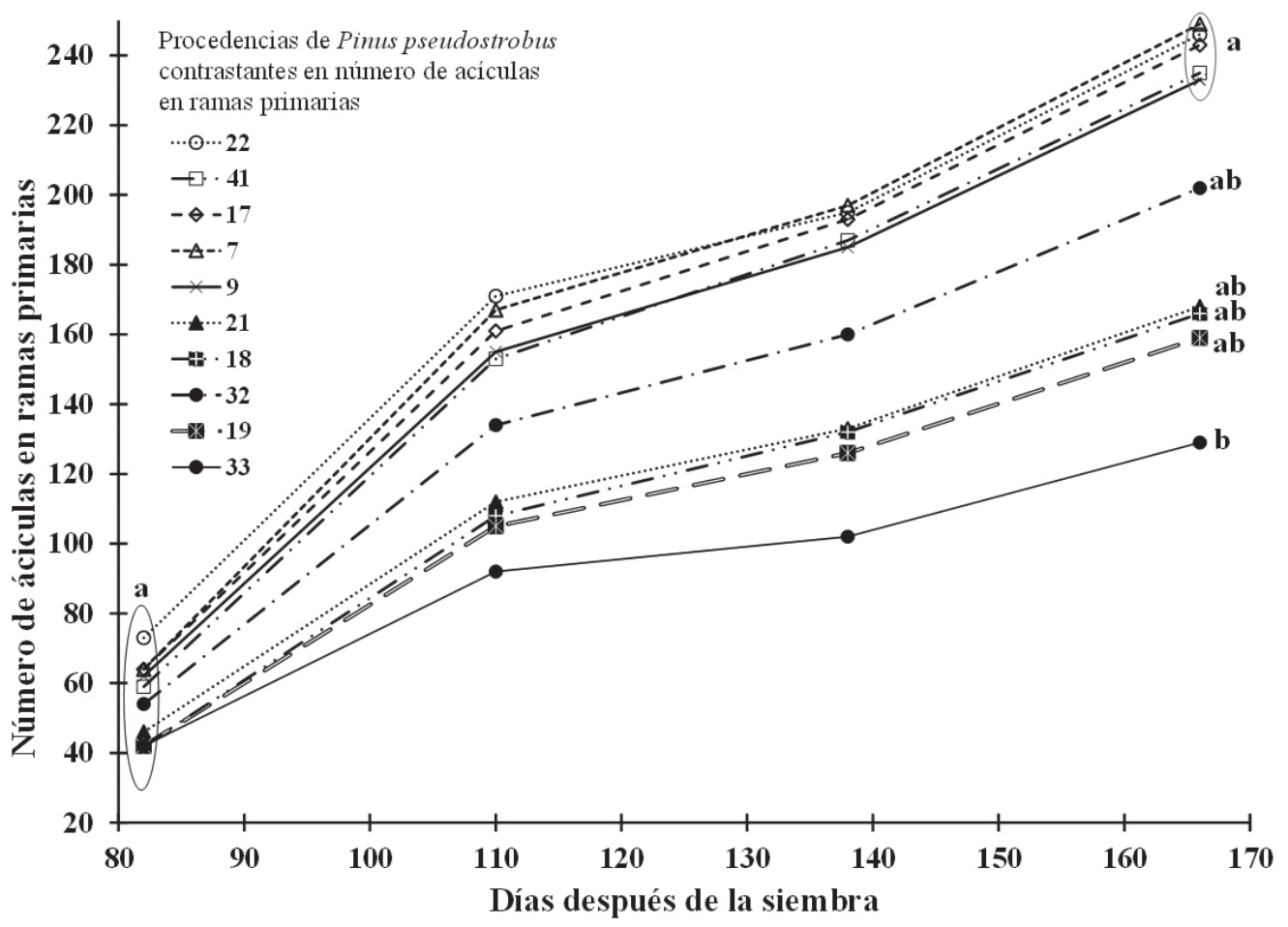

(a)

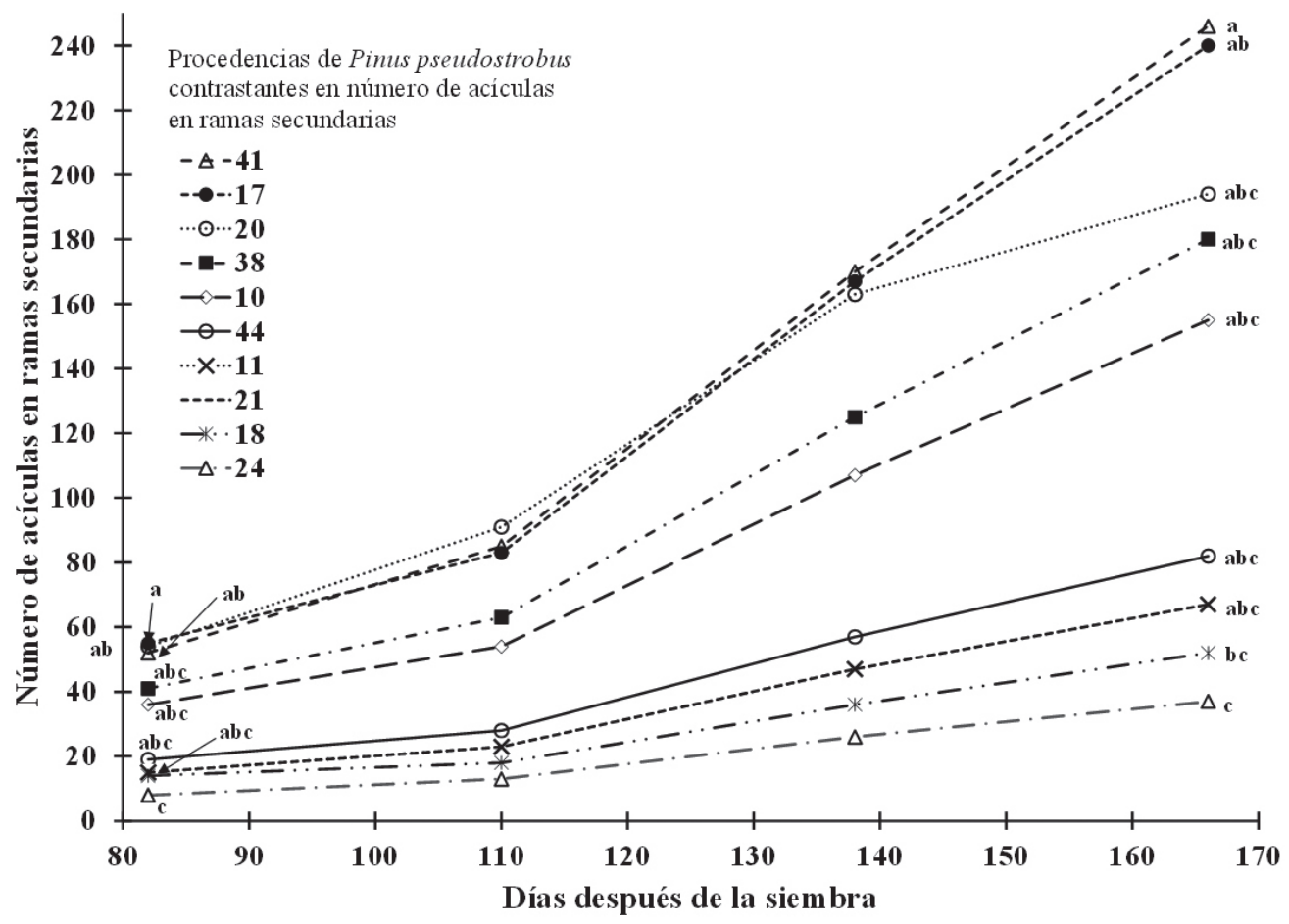

(b)

FiguRA 3. Patrones de crecimiento en relación a formación de braquiblastos en ramillas primarias (a) y secundarias (b) de 10 procedencias/ progenitores de Pinus pseudostrobus con diferente tasa de crecimiento en vivero. Promedios con letras iguales no difieren estadísticamente (Tukey, $\mathrm{P}<0.05)$.

FiguRE 3. Patterns of growth in number of needles on primary (a) and secondary (b) branches of 10 provenances/parents of Pinus pseudostrobus progenitors with different growth rate in nursery. Averages with similar letter are not statistically different $($ Tukey, $\mathrm{P}<0.05)$. 
$\mathrm{cm})$. Un patrón semejante se observó en las procedencias de menor crecimiento a 166 dds $(12,8$ a $14,0 \mathrm{~cm})$, excepto en 35 que también presentó menor crecimiento en 166 dds $(13,4 \mathrm{~cm})$. Las diferencias entre el crecimiento a 54 y 166 dds determinó que las procedencias $1,2,3,9,17$, $24,25,28,34,36$ y 38 , efectivamente presentaron un mayor crecimiento de 1,3 a $1,5 \mathrm{~mm} /$ día. Estas diferencias también se confirman con la tasa de crecimiento relativo. En contraposición, en diámetro de tallo las procedencias de mayor crecimiento fueron 3,17 , y 25 (2,78 a 2,81 $\mathrm{mm}$, Tabla III). Demostrando que solo la procedencia 17 coincidió con el mejor crecimiento en las dos variables.

En relación a la generación de braquiblastos, a 82 dds no hubo diferencias entre procedencias para el número de braquiblastos sobre ramillas primarias. Sin embargo, la velocidad de crecimiento a partir de ese estado fue exponencial a razón de un promedio de 1,4 a 2,2 braquiblastos por día. En este sentido las procedencias con mayor tasa de aparición de braquiblastos sobre ramillas secundarias fueron $6,7,9,22,41,44$ y 49 con promedio mínimo de 2 braquiblastos por día, y correspondieron a las que presentaron mayor número de braquiblastos a 166 dds. En términos de número de braquiblastos sobre ramillas secundarias, las diferencias no fueron tan marcadas excepto para las procedencias 17 y 41 (240 a 246 braquiblastos). En contraposición, las procedencias 18 y 24 presentaron 52 y 37 braquiblastos sobre ramillas primarias, respectivamente. La capacidad de producir braquiblastos en la planta facilita mayor capacidad fotosintética porque además de poseer mayor área foliar también posee tallos verdes que pueden realizar actividades de fotosíntesis. A 166 dds se evaluó la sobrevivencia de plantas en vivero, la que estuvo relacionada directamente con el número de plantas emergidas, es decir no hubo plántulas muertas, lo que determinó que la sobrevivencia en vivero fuera de 9,5 a $73,4 \%$, lo que proporciona un estimador que ayuda a orientar los programas de propagación de P. pseudostrobus de la Sierra Norte de Oaxaca, en vivero (Tabla IV).

TABLA II. Significancia de cuadrados medios del análisis de varianza en variables asociadas al crecimiento de plantas de Pinus pseudostrobus en vivero, transcurridos 54, 82 y 166 días después de la siembra.

TABLE II. Significance of mean squares of analysis of variance in variables associated with the growth of Pinus pseudostrobus in nursery, at 54,82 and 166 days after planting.

\begin{tabular}{|c|c|c|c|c|c|}
\hline \multirow[t]{2}{*}{ VARIABLES EVALUADAS } & \multicolumn{4}{|c|}{ FUENTES DE VARIACIÓN } & \multirow{2}{*}{$\begin{array}{c}\text { COEF. DE } \\
\text { VARIACIÓN (\%) }\end{array}$} \\
\hline & REP. & Procedencias & $\begin{array}{c}\text { Plantas } \\
(\text { Proced. })^{\dagger}\end{array}$ & ERROR & \\
\hline \multicolumn{6}{|c|}{ CRECIMIENTO A 54 DÍAS DESPUÉS DE LA SIEMBRA: } \\
\hline Altura de planta & $0,639^{\mathrm{NS}}$ & $1,185 * *$ & $0,289^{\mathrm{NS}}$ & 0,351 & 15,5 \\
\hline Diámetro basal de tallo & $0,038^{\mathrm{NS}}$ & $0,062 * *$ & $0,014^{\mathrm{NS}}$ & 0,023 & 13,4 \\
\hline
\end{tabular}

CRecimiento EN NÚMERo de aCícula EN RAMAS a 82 días DESPUÉS DE LA SIEMBRA:

\begin{tabular}{llllll}
\hline Ramas primarias & $0,757^{\mathrm{NS}}$ & $1,504^{\mathrm{NS}}$ & $0,500^{\mathrm{NS}}$ & 1,169 & 14,6 \\
Ramas secundarias & $25,742^{*}$ & $6,149^{*}$ & $2,227^{\mathrm{NS}}$ & 3,217 & 36,2 \\
\hline
\end{tabular}

CRecimiento a 166 díAs Después de LA SIEMBRA:

\begin{tabular}{|c|c|c|c|c|c|}
\hline Altura de planta & $128,372 * *$ & $45,208 * *$ & $6,414^{\mathrm{NS}}$ & 9,759 & 18,7 \\
\hline Diámetro basal de tallo & $3,251 * *$ & $0,307 * *$ & $0,136^{\mathrm{NS}}$ & 0,157 & 15,8 \\
\hline Braquiblastos en ramas primarias & $20,521^{* *}$ & $7,841^{*}$ & $3,151^{\mathrm{NS}}$ & 4,677 & 15,3 \\
\hline Braquiblastos en ramas secundarias & $73,644 * *$ & $28,101 * *$ & $4,700^{\mathrm{NS}}$ & 11,613 & 33,3 \\
\hline Emergencia y sobrevivencia & $0,070^{\mathrm{NS}}$ & $0,091 * *$ & -- & 0,018 & 18,1 \\
\hline $\mathrm{TCR}^{\dagger \dagger}$ & $0,157 *$ & $0,060 * *$ & $0,012^{\mathrm{NS}}$ & 7,280 & 9,0 \\
\hline
\end{tabular}

*significativo a $\mathrm{P}<0,05 ; * *$ significativo a $\mathrm{P}<0,01 ;{ }^{\dagger}$ plantas anidadas en procedencias, de acuerdo con el modelo líneal de análisis establecido; ${ }^{\dagger} \mathrm{TCR}$ = tasa de crecimiento relativo en relación a altura de planta entre 54 y 166 días después de la siembra./*significant at $\mathrm{P}<0.05$; ** significant at $\mathrm{P}<0.01$; †plants nested in provenances, according to lineal model of analysis stated; $\dagger \dagger \mathrm{TCR}=$ relative growth rate for plant height between 54 and 166 days after planting. 
Gayana Bot. 73(1), 2016

TABla III. Promedios de altura de planta, diámetro de tallo y tasa de crecimiento en Pinus pseudostrobus a 54 y 166 días después de la siembra.

TABLE III. Average of plant height, stem diameter and growth rate in Pinus pseudostrobus at 54 and 166 days after planting.

\begin{tabular}{|c|c|c|c|c|c|c|}
\hline \multirow[t]{2}{*}{ Procedencias } & \multicolumn{2}{|c|}{ Altura De PLANTA $(\mathrm{cm})$} & \multirow{2}{*}{$\begin{array}{l}\text { CREC./ DÍA } \\
(\mathrm{mm} / \text { día })\end{array}$} & \multicolumn{2}{|c|}{ DiÁMETRO DE TALLO (mm) } & \multirow{2}{*}{$\begin{array}{c}\mathrm{TCR}^{\dagger \dagger}(\mathrm{cm} x \\
\text { 100/día) }\end{array}$} \\
\hline & $54 \mathrm{dds}$ & $166 \mathrm{dds}$ & & $54 \mathrm{dds}$ & 166 dds & \\
\hline 1 & 3,9 abcde $^{*}$ & 20,1 a & 1,5 & $1,18 \mathrm{abc}$ & $2,46 \mathrm{abc}$ & $1,68 \mathrm{a}$ \\
\hline 2 & 4,1 abcd & $19,1 \mathrm{ab}$ & 1,3 & $1,25 \mathrm{ab}$ & $2,61 \mathrm{abc}$ & $1,68 \mathrm{a}$ \\
\hline 3 & 3,7 abcde & $18,1 \mathrm{abc}$ & 1,3 & $1,15 \mathrm{abc}$ & $2,81 \mathrm{a}$ & $1,62 \mathrm{abc}$ \\
\hline 5 & 3,7 abcde & 15,7 abcde & 1,1 & $1,21 \mathrm{abc}$ & $2,33 \mathrm{abc}$ & $1,56 \mathrm{abc}$ \\
\hline 6 & 3,4 cde & 15,5 bcde & 1,1 & $1,21 \mathrm{abc}$ & $2,47 \mathrm{abc}$ & $1,53 \mathrm{abc}$ \\
\hline 7 & 3,8 abcde & 16,4 abcde & 1,1 & $1,17 \mathrm{abc}$ & $2,51 \mathrm{abc}$ & $1,57 \mathrm{abc}$ \\
\hline 8 & 3,7 abcde & 14,8 bcde & 1,0 & $1,20 \mathrm{abc}$ & $2,23 \mathrm{c}$ & $1,52 \mathrm{abc}$ \\
\hline 9 & 4,1 abcd & $18,7 \mathrm{ab}$ & 1,3 & $1,11 \mathrm{abc}$ & $2,67 \mathrm{abc}$ & $1,67 \mathrm{ab}$ \\
\hline 10 & 3,7 abcde & 15,9 abcde & 1,1 & $1,09 \mathrm{abc}$ & $2,54 \mathrm{abc}$ & $1,57 \mathrm{abc}$ \\
\hline 11 & 3,4 cde & 16,1 abcde & 1,1 & $1,07 \mathrm{abc}$ & $2,59 \mathrm{abc}$ & $1,54 \mathrm{abc}$ \\
\hline 12 & 3,7 abcde & $14,0 \mathrm{cde}$ & 0,9 & $1,20 \mathrm{abc}$ & $2,42 \mathrm{abc}$ & $1,52 \mathrm{abc}$ \\
\hline 13 & $4,5 \mathrm{a}$ & 17,3 abcde & 1,2 & $1,19 \mathrm{abc}$ & $2,41 \mathrm{abc}$ & $1,66 \mathrm{ab}$ \\
\hline 15 & 4,0 abcdde & $17,5 \mathrm{abcd}$ & 1,2 & $1,11 \mathrm{abc}$ & $2,42 \mathrm{abc}$ & $1,64 \mathrm{abc}$ \\
\hline 16 & 3,7 abcde & 16,5 abcde & 1,1 & $1,13 \mathrm{abc}$ & $2,46 \mathrm{abc}$ & $1,58 \mathrm{abc}$ \\
\hline 17 & 4,2 abcd & $19,1 \mathrm{ab}$ & 1,3 & $1,21 \mathrm{abc}$ & $2,79 \mathrm{ab}$ & $1,69 \mathrm{a}$ \\
\hline 18 & 3,2 de & 16,1 abcde & 1,1 & 0,99 c & $2,31 \mathrm{bc}$ & $1,52 \mathrm{abc}$ \\
\hline 19 & 3,6 abcde & 16,1 abcde & 1,1 & $1,08 \mathrm{abc}$ & $2,44 \mathrm{abc}$ & $1,53 \mathrm{abc}$ \\
\hline 20 & 3,7 abcde & 14,7 bcde & 1,0 & $1,13 \mathrm{abc}$ & $2,48 \mathrm{abc}$ & $1,60 \mathrm{abc}$ \\
\hline 21 & 3,6 abcde & 14,7 bcde & 1,0 & $1,11 \mathrm{abc}$ & $2,52 \mathrm{abc}$ & $1,53 \mathrm{abc}$ \\
\hline 22 & 3,9 abcde & $17,8 \mathrm{abcd}$ & 1,2 & $1,27 \mathrm{a}$ & $2,58 \mathrm{abc}$ & $1,64 \mathrm{abc}$ \\
\hline 23 & 3,5 bcde & $12,8 \mathrm{e}$ & 0,8 & $1,10 \mathrm{abc}$ & $2,34 \mathrm{abc}$ & $1,46 \mathrm{bc}$ \\
\hline 24 & 3,5 bcde & $17,9 \mathrm{abcd}$ & 1,3 & $1,07 \mathrm{abc}$ & $2,41 \mathrm{abc}$ & $1,60 \mathrm{abc}$ \\
\hline 25 & $4,4 \mathrm{ab}$ & $18,5 \mathrm{abc}$ & 1,3 & $1,11 \mathrm{abc}$ & $2,79 \mathrm{ab}$ & $1,69 \mathrm{a}$ \\
\hline 28 & 4,0 abcde & $19,0 \mathrm{ab}$ & 1,3 & $1,03 \mathrm{bc}$ & $2,56 \mathrm{abc}$ & $1,66 \mathrm{ab}$ \\
\hline 29 & 3,8 abcde & 14,7 bcde & 1,0 & $1,03 \mathrm{bc}$ & $2,46 \mathrm{abc}$ & $1,55 \mathrm{abc}$ \\
\hline 32 & $4,3 \mathrm{abc}$ & $18,1 \mathrm{abc}$ & 1,2 & $1,18 \mathrm{abc}$ & $2,59 \mathrm{abc}$ & $1,63 \mathrm{abc}$ \\
\hline 33 & 3,5 bcde & 14,0 cde & 0,9 & $1,07 \mathrm{abc}$ & $2,51 \mathrm{abc}$ & $1,44 \mathrm{c}$ \\
\hline 34 & 3,9 abcde & $18,0 \mathrm{abcd}$ & 1,3 & $1,07 \mathrm{abc}$ & $2,53 \mathrm{abc}$ & $1,64 \mathrm{abc}$ \\
\hline 35 & $3,1 \mathrm{e}$ & 13,4 de & 0,9 & $1,22 \mathrm{abc}$ & $2,37 \mathrm{abc}$ & $1,57 \mathrm{abc}$ \\
\hline 36 & 3,7 abcde & $18,1 \mathrm{abc}$ & 1,3 & $1,03 \mathrm{bc}$ & $2,30 \mathrm{bc}$ & $1,61 \mathrm{abc}$ \\
\hline 37 & 3,6 abcde & 14,6 bcde & 1,0 & $1,11 \mathrm{abc}$ & $2,48 \mathrm{abc}$ & $1,51 \mathrm{abc}$ \\
\hline 38 & 3,7 abcde & $19,0 \mathrm{ab}$ & 1,4 & $1,11 \mathrm{abc}$ & $2,71 \mathrm{abc}$ & $1,64 \mathrm{abc}$ \\
\hline 41 & 3,7 abcde & 16,3 abcde & 1,1 & $1,09 \mathrm{abc}$ & $2,62 \mathrm{abc}$ & $1,58 \mathrm{abc}$ \\
\hline 42 & 4,1 abcd & 17,0 abcde & 1,2 & $1,15 \mathrm{abc}$ & $2,63 \mathrm{abc}$ & $1,63 \mathrm{abc}$ \\
\hline 44 & 4,0 abcdde & 17,4 abcde & 1,2 & $1,15 \mathrm{abc}$ & $2,37 \mathrm{abc}$ & $1,64 \mathrm{abc}$ \\
\hline 48 & 4,0 abcdde & 16,7 abcde & 1,1 & $1,11 \mathrm{abc}$ & $2,27 \mathrm{c}$ & $1,61 \mathrm{abc}$ \\
\hline 49 & 4,0 abcdde & 14,9 bcde & 1,0 & $1,14 \mathrm{abcd}$ & $2,35 \mathrm{abc}$ & $1,68 \mathrm{abc}$ \\
\hline 52 & 3,7 abcde & 16,7 abcde & 1,2 & $1,02 \mathrm{c}$ & $2,41 \mathrm{abc}$ & $1,59 \mathrm{abc}$ \\
\hline Promedio & 3,7 & 16,6 & 1,1 & 1,13 & 2,49 & 1,59 \\
\hline
\end{tabular}

dds, días después de la siembra; TCR, tasa de crecimiento relativo en altura de planta entre 54 y 166 días después de la siembra; ‘en columna, medias con diferente letra son estadísticamente diferentes (Tukey, $\mathrm{P}<0,05$ )./ dds, days after planting; TCR $=$ relative growth rate for plant height between 54 and 166 days after planting; "in column, means with different letter are statistically different $($ Tukey, $\mathrm{P}<0.05$ ). 
Variación del crecimiento en vivero de Pinus pseudostrobus: VILLEGAS-JiMÉnEZ, D.E. ET AL.

TABLA IV. Promedio de braquiblastos en ramillas primarias y secundarias a 82 y 166 días después de la siembra, y sobrevivencia de plantas de Pinus pseudostrobus a 54 y 166 días después de la siembra.

TABLE IV. Average number of brachyblasts on primary and secondary branches at 82 and 166 days after planting, and survival of Pinus pseudostrobus plants from 54 to 166 days after planting.

\begin{tabular}{|c|c|c|c|c|c|c|c|}
\hline \multirow[t]{2}{*}{ Procedencias } & \multicolumn{3}{|c|}{$\begin{array}{c}\text { NÚMERO DE BRAQUIBLASTOS EN RAMILLAS } \\
\text { PRIMARIAS }\end{array}$} & \multicolumn{3}{|c|}{$\begin{array}{l}\text { NÚMERO DE BRAQUIBLASTOS EN RAMILLAS } \\
\text { SECUNDARIAS }\end{array}$} & \multirow{2}{*}{$\begin{array}{c}\text { SOBREVIVENCIA } \\
\text { DESDE } 54 \text { A } 166 \\
\text { dds }(\%)\end{array}$} \\
\hline & $82 \mathrm{dds}^{\dagger}$ & $166 \mathrm{dds}$ & Prom./ día & 82 dds & $166 \mathrm{dds}$ & Prom./ día & \\
\hline 1 & $55 \mathrm{a}^{\ddagger}$ & $203 \mathrm{ab}$ & 1,76 & $19 a b c$ & $89 \mathrm{abc}$ & 0,83 & $63,3 \mathrm{ab}$ \\
\hline 2 & $63 \mathrm{a}$ & $220 \mathrm{ab}$ & 1,87 & $45 a b c$ & $144 a b c$ & 1,18 & $63,9 a b$ \\
\hline 3 & $58 \mathrm{a}$ & $194 \mathrm{ab}$ & 1,62 & $28 a b c$ & $201 \mathrm{abc}$ & 2,06 & 28,9 bcde \\
\hline 5 & $50 \mathrm{a}$ & $191 \mathrm{ab}$ & 1,68 & $49 a b c$ & $68 \mathrm{abc}$ & 0,23 & 31,6 abcde \\
\hline 6 & $59 \mathrm{a}$ & $229 a b$ & 2,02 & $22 \mathrm{abc}$ & $117 \mathrm{abc}$ & 1,13 & $45,5 \mathrm{abcd}$ \\
\hline 7 & $64 \mathrm{a}$ & $249 a$ & 2,20 & $32 \mathrm{abc}$ & $120 \mathrm{abc}$ & 1,05 & $51,6 \mathrm{abc}$ \\
\hline 8 & $51 \mathrm{a}$ & $169 \mathrm{ab}$ & 1,40 & $26 a b c$ & $83 \mathrm{abc}$ & 0,68 & 40,8 abcde \\
\hline 9 & $61 \mathrm{a}$ & $233 a b$ & 2,05 & $18 a b c$ & $78 \mathrm{abc}$ & 0,71 & 48,6 abcd \\
\hline 10 & $57 \mathrm{a}$ & $218 \mathrm{ab}$ & 1,92 & $36 a b c$ & $155 a b c$ & 1,42 & 34,0 abcde \\
\hline 11 & $56 \mathrm{a}$ & $213 a b$ & 1,87 & $15 a b c$ & $67 \mathrm{abc}$ & 0,62 & 57,8 ab \\
\hline 12 & $62 \mathrm{a}$ & $207 \mathrm{ab}$ & 1,73 & $21 \mathrm{abc}$ & $91 \mathrm{abc}$ & 0,83 & 27,8 bcde \\
\hline 13 & $52 \mathrm{a}$ & $161 \mathrm{ab}$ & 1,30 & $25 \mathrm{abc}$ & $142 \mathrm{abc}$ & 1,39 & $60,5 \mathrm{ab}$ \\
\hline 15 & $50 \mathrm{a}$ & $180 \mathrm{ab}$ & 1,55 & $25 \mathrm{abc}$ & $143 a b c$ & 1,40 & 23,5 abcde \\
\hline 16 & $55 \mathrm{a}$ & $194 \mathrm{ab}$ & 1,65 & $30 \mathrm{abc}$ & $155 \mathrm{abc}$ & 1,49 & 48,9 abcd \\
\hline 17 & $64 \mathrm{a}$ & $243 a$ & 2,13 & 55 a & $240 \mathrm{ab}$ & 2,20 & $58,8 \mathrm{ab}$ \\
\hline 18 & $42 \mathrm{a}$ & $166 \mathrm{ab}$ & 1,48 & $14 \mathrm{abc}$ & $52 \mathrm{bc}$ & 0,45 & $48,6 \mathrm{abcd}$ \\
\hline 19 & $42 \mathrm{a}$ & $159 \mathrm{ab}$ & 1,39 & $18 a b c$ & $78 \mathrm{abc}$ & 0,71 & 36,0 abcde \\
\hline 20 & $48 \mathrm{a}$ & $215 \mathrm{ab}$ & 1,99 & $54 \mathrm{ab}$ & $194 \mathrm{abc}$ & 1.67 & $9,5 \mathrm{e}$ \\
\hline 21 & $46 \mathrm{a}$ & $168 \mathrm{ab}$ & 1,45 & $13 \mathrm{abc}$ & $71 \mathrm{abc}$ & 0,69 & 26,5 bcde \\
\hline 22 & $73 \mathrm{a}$ & 246 a & 2,06 & $21 \mathrm{abc}$ & $146 \mathrm{abc}$ & 1,49 & $65,3 \mathrm{ab}$ \\
\hline 23 & $54 \mathrm{a}$ & $204 \mathrm{ab}$ & 1,79 & $25 \mathrm{abc}$ & $98 \mathrm{abc}$ & 0,87 & $14,9 \mathrm{cde}$ \\
\hline 24 & $57 \mathrm{a}$ & $217 \mathrm{ab}$ & 1,90 & $8 \mathrm{c}$ & $37 \mathrm{c}$ & 0,35 & $61,2 \mathrm{ab}$ \\
\hline 25 & $51 \mathrm{a}$ & $195 \mathrm{ab}$ & 1,71 & $16 \mathrm{abc}$ & $72 a b c$ & 0,67 & 44,5 abcde \\
\hline 28 & $65 \mathrm{a}$ & $225 \mathrm{ab}$ & 1,90 & $11 \mathrm{abc}$ & $68 \mathrm{abc}$ & 0,68 & 70,4 ab \\
\hline 29 & $57 \mathrm{a}$ & $212 \mathrm{ab}$ & 1,85 & $13 \mathrm{abc}$ & $97 \mathrm{abc}$ & 1,00 & 30,1 abcde \\
\hline 32 & $54 \mathrm{a}$ & $202 \mathrm{ab}$ & 1,76 & $32 \mathrm{abc}$ & $137 \mathrm{abc}$ & 1,25 & $60,2 \mathrm{ab}$ \\
\hline 33 & $42 \mathrm{a}$ & $129 \mathrm{~b}$ & 1,04 & $36 \mathrm{abc}$ & $162 a b c$ & 1,50 & 39,4 abcde \\
\hline 34 & $59 \mathrm{a}$ & $213 \mathrm{ab}$ & 1,83 & $24 \mathrm{abc}$ & $94 \mathrm{abc}$ & 0,83 & 45,2 abcde \\
\hline 35 & $50 \mathrm{a}$ & $214 \mathrm{ab}$ & 1,95 & $31 \mathrm{abc}$ & $157 \mathrm{abc}$ & 1,50 & 11,5 de \\
\hline 36 & $58 \mathrm{a}$ & $219 a b$ & 1,92 & $16 \mathrm{abc}$ & $78 \mathrm{abc}$ & 0,74 & $63,2 \mathrm{ab}$ \\
\hline 37 & $55 \mathrm{a}$ & $221 \mathrm{ab}$ & 1,98 & $28 \mathrm{abc}$ & $121 \mathrm{abc}$ & 1,11 & $57,4 \mathrm{abc}$ \\
\hline 38 & $60 \mathrm{a}$ & $228 \mathrm{ab}$ & 2,00 & $41 \mathrm{abc}$ & $180 \mathrm{abc}$ & 1,65 & 73,4 a \\
\hline 41 & 59 a & $235 \mathrm{a}$ & 2,10 & $52 a b$ & 246 a & 2,31 & $54,7 \mathrm{abc}$ \\
\hline 42 & $58 \mathrm{a}$ & $174 \mathrm{ab}$ & 1,38 & $33 \mathrm{abc}$ & $96 \mathrm{abc}$ & 0,75 & 67,6 ab \\
\hline 44 & $59 \mathrm{a}$ & 244 a & 2,20 & $19 a b c$ & $82 a b c$ & 0,75 & $46,5 \mathrm{abcd}$ \\
\hline 48 & $50 \mathrm{a}$ & $187 \mathrm{ab}$ & 1,63 & $18 a b c$ & $92 \mathrm{abc}$ & 0,88 & 42,8 abcde \\
\hline 49 & $54 \mathrm{a}$ & $229 a b$ & 2,08 & $37 \mathrm{abc}$ & $131 \mathrm{abc}$ & 1,12 & 40,1 abcde \\
\hline 52 & $57 \mathrm{a}$ & $203 \mathrm{ab}$ & 1,74 & $51 \mathrm{abc}$ & $101 \mathrm{abc}$ & 0,60 & 38,7 abcde \\
\hline Promedio & 55.4 & 205.5 & 1,79 & 27,8 & 117,9 & 1,07 & 45,61 \\
\hline
\end{tabular}

†dds, días después de la siembra; *en columna, medias con diferente letra son estadísticamente diferentes (Tukey, P $<0,05) . /{ }^{\dagger}$ dds, days after planting; $\$$ in column, means with different letter are statistically different (Tukey, $\mathrm{P}<0.05$ ). 


\section{DISCUSIÓN}

Las procedencias o progenitores evaluados se localizan en la Sierra Norte de Oaxaca, y presentaban fenotipos que fueron considerados promisorios por los productores locales. La descripción preliminar de los progenitores en campo mostró una alta variabilidad en caracteres descriptivos dasométricos maderables (Tabla I). Esta variación de campo, también se reflejó en la evaluación de plántulas en vivero. La sobrevivencia varió de 9,5 a 73,4\% (Tabla IV), aun cuando se pueden considerar valores bajos fueron mayores a los porcentajes de germinación reportados por Andersen et al. (2008) para diferentes procedencias de Abies guatemalensis Rehder en Guatemala, la que varió de 0,8 a $14,5 \%$ de germinación y una vez germinado obtuvo una sobrevivencia de 61,5 a $87,6 \%$. En Pinus teocote Schltdl. \& Cham., Ramírez-García et al. (2001) determinaron diferencias significativas entre procedencias en germinación de semilla desde 3 a 95\%. Esto indica que la baja sobrevivencia encontrada en este trabajo no es exclusiva de P. pseudostrobus. En la cinética de crecimiento en altura de planta e incremento en braquiblastos se observaron diferencias entre procedencias. En este caso las procedencias 9 y 17, consistentemente presentaron el mayor número de braquiblastos y altura de planta de 54 a 166 días después de la siembra (dds).

Las diferencias entre procedencia en relación a germinación y sobrevivencia de plantas, y crecimiento inicial obedece, en parte, a diferencias morfológicas y reservas en las semillas colectadas. En este sentido, Hernández-Carmona et al. (2003) determinaron diferencias significativas entre procedencias de semilla de $P$. pseudostrobus de Michoacán, Guerrero y Oaxaca, México, y Chimaltenango, Guatemala, principalmente en peso, ancho y largo de semilla. También es de resaltar que el crecimiento de plántulas en los primeros días después de la emergencia tiene como base las reservas en la semilla hasta que inicia la captura de luz a través de hojas o acículas (Villar et al. 2004).

Entre procedencias evaluadas se detectaron diferencias significativas $(p<0.05)$, en altura de planta tanto a 54 como 166 dds. No hubo un patrón, entre las procedencias con mayor altura de planta, a $166 \mathrm{dds}$, con origen de mayor o menor altitud. Estos patrones de crecimiento diferencial de procedencias son semejantes a los evaluados por Castellanos-Acuña et al. (2013) en P. pseudostrobus en plantas de 3 y 5 meses de edad en vivero, y homologa a la reportada por Viveros-Viveros et al. (2005b) en la misma especie pero en una plantación en campo a 15 y 24 meses de edad de las plantas, en Los Amoles, Michoacán. El mismo patrón se evaluó también en diámetro basal del tallo, la variación fue de 2,2 a 2,8 $\mathrm{mm}$ a 166 dds (Tabla III).

En relación al número de braquiblastos sobre ramillas primarias y secundarias, además de alta variabilidad entre procedencia, la tasa de aparición de braquiblastos fue de 1 a 2 braquiblastos sobre ramillas primarias y secundarias por día en un intervalo de 54 a 166 días después de la siembra (Tabla IV). Esto indica que las plántulas estaban en una fase de crecimiento exponencial como lo denota Villar et al. (2004) en especies leñosas, siempre que no se encuentran en condiciones de estrés hídrico principalmente. La alta tasa de crecimiento inicial en altura y número de braquiblastos en $P$. pseudostrobus le provee de mayor capacidad adaptativa al terreno donde se realizara la plantación, y en este sentido Sáenz et al. (2011) considera a esta especie como una de las especies de pinos con la mayor tasa de crecimiento inicial y muy útil para su establecimiento en campo.

En Pinus oocarpa Schiede, Viveros-Viveros et al. (2005b) demostraron que en altura de planta y diámetro basal del tallo la especie presentó baja heredabilidad en ambos caracteres $\left(0,27<\mathrm{h}^{2}<0,40\right)$, en plantas de 2 a 6 meses de edad en vivero. No obstante, los caracteres pueden ser útiles en la selección temprana de progenitores promisorios como lo propone Farfán et al. (2002) para P. ayacahuite Ehren. En este sentido las procedencias promisorias en esta evaluación fueron: 9, 17, 22 y 41, las que se originaron de árboles progenitores de $P$. pseudostrobus ubicados en altitudes de 2.670 a $2.930 \mathrm{~m}$ de los bosques de la Sierra Norte de Oaxaca.

\section{CONCLUSIONES}

Se determinaron diferencias significativas entre procedencias de $P$. pseudostrobus de la Sierra Norte de Oaxaca, en germinación y sobrevivencia, crecimiento de planta, diámetro basal de tallo, número de ramas primarias y secundarias y tasa de crecimiento relativo. En la evaluación se manifestó un crecimiento exponencial de plantas en vivero, un promedio de 0,8 a $1,5 \mathrm{~mm}$ de altura por día y un promedio de 1 a 2 braquiblastos sobre ramillas primarias y secundarias por día en un intervalo de 82 a 166 días después de la siembra. Las procedencias promisorias en esta evaluación fueron: 9,17 , 22 y 41, las que se originaron de árboles progenitores de $P$. pseudostrobus ubicados en altitudes de 2.670 a $2.930 \mathrm{~m}$.

\section{BIBLIOGRAFÍA}

Andersen, U.S., J.P. Prado C., U.B. Nielsen \& J. Kollmann. 2008. Provenance variation in germination and seedling growth of Abies guatemalensis Rehder. Forest Ecology and Management 255: 1831-1840.

Cambrón, V.H., H. Suzán, J.J. Vargas, N.M. Sánchez \& C. SáenzROMERo. 2013. Estrategias de crecimiento y distribución de biomasa en Pinus pseudostrobus bajo diferentes condiciones de competencia. Revista Fitotecnia Mexicana 36(1): 71-79.

Castellanos-Acuña, D., C. SÁenz-Romero, R.A. LindingCisneros, N.M. Sánchez-Vargas, P. Lobbit \& J.C. 
Variación del crecimiento en vivero de Pinus pseudostrobus: VILLEGAS-JiMÉnez, D.E. ET AL.

Montero-CAstro. 2013. Variación altitudinal entre especies y procedencias de Pinus pseudostrobus, $P$. devoniana y $P$. leiophylla. Ensayo de vivero. Revista Chapingo Serie Ciencias Forestales y del Ambiente 19(3): 399-411.

Delgado, P., J. NúÑez, M.C. Rocha \& H.J. MuÑoz. 2013. Variación genética de dos áreas semilleras de pino establecidas en el Estado de Michoacán. Revista Mexicana de Ciencias Forestales 4(18): 104-115.

Delgado, P., \& D. Piñeiro. 2002. Sistemática filogeográfica y sus aplicaciones a la evolución y conservación de los bosques de coníferas en México: El caso de Pinus montezumae y $P$. pseudostrobus. Acta Universitaria 12(2): 3-19.

Delgado, P., R. Salas-Lizana, A. Vázquez-Lobo, A. Wegier, M. Anzidei, E.R. Alvares-Buylla, G.G. Vendramin \& D. PiñeIro. 2007. Introgresive hybridization in Pinus montezumae Lamb and Pinus pesudostrobus Lindl. (Pinaceae): morphological and molecual (cpSSR) evidence. International Journal of Plant Science 168(6): 861-876.

Díaz-Gallegos, J.R., J-F. Mas \& A. Velázquez-Montes. 2008. Monitoreo de los patrones de deforestación en el corredor biológico Mesoamericano, México. Interciencia 33(12): 882-890.

Equiluz, T. 1982. Clima y distribución del genero Pinus en México. Revista Ciencia Forestal 38(7): 30-44.

Farfán, E.G., J. JASSO, J. López, J.J. Vargas \& C. Ramírez. 2002. Parámetros genéticos y eficiencia de la selección temprana en Pinus ayacahuite Ehren. var. ayacahuite. Revista Fitotecnia Mexicana 25(3): 239-246.

Hernández-Carmona, O., E.O. Ramírez-García \& L. Mendizábal-Hernández. 2003. Variación en semillas de cinco procedencias de Pinus pseudostrobus Lindl. Foresta Veracruzana 5(2): 23-28.

Muñoz, H.H., G. Orozco, V.M. Coria, Y.Y. MuÑoz \& J. García. 2011. Manejo de área semillera de Pinus pseudostrobus Lindl. y Abies religiosa (H.B.K.) Schltdl. et Cham. y selección de árboles superiores en Michoacán, México. Foresta Veracruzana 12(2): 29-36.

Palacio-Prieto, J.L., G. Bocco, A. Velázquez, J-F. Mas, F. TAKaki-Takaki，A. Victoria， L. Luna-GonzÁlez，G. Gómez-Rodríguez, J. López-García，M. Palma-Muñoz, I. Trejo-Vázquez, A. Peralta-Higuera, J. Prado-Molina, A. Rodríguez-Aguilar, R. Mayorga-SAucedo \& F. GonzÁlez-Medrano. 2000. La condición actual de los recursos forestales en México: resultados del inventario forestal nacional 2000. Investigaciones Geográficas, Boletín del Instituto de Geografía-UNAM 43: 183-203.

Ramírez-García, E.O., J. Alba-Landa \& L.C. MendizÁbalHERNÁNDEZ. 2001. Evaluación en vivero de un ensayo de procedencias/progenie de Pinus teocote Schl. \& Cham. Foresta Veracruzana 3(1): 27-34.

Reyes, R., N. Viveros \& V. Pérez. 1995. Resistencia natural de trece maderas mexicanas al ataque de termitas subterráneas. Madera y Bosques 1(1): 39-47.

SÁEnZ, J.T., H.J. MuÑoz \& A. RuedA. 2011. Especies promisorias de clima templado para plantaciones forestales comerciales en Michoacán. Libro Técnico Núm. 10. SAGARPA-
INIFAP-CIRPAC-Campo Experimental Uruapan. Uruapan, Michoacán. 213 p.

Sáenz-Romero, C., A.E. Snively \& R. Linding-Cisneros. 2003. Conservation and restoration of pine forest genetic resources in Mexico. Silvae Genetica 52(5-6): 233-237

Sáenz-Romero, C., G. E. Rehfeldt, J.C. Soto-Correa, S. AguilarAguilar, V. Zamarripa-Morales \& J. López-Upton. 2012. Altitudinal genetic variation among Pinus pseudostrobus populations from Michoacán, Mexico, two locations shadehouse test results. Revista Fitotecnia Mexicana 35(2): 111-120.

SAhagún, C. J. 1998. Construcción de análisis de los modelos fijos, aleatorios y mixtos. Boletín Técnico No. 2, Universidad Autónoma de Chapingo Departamento de Fitotecnia, Programa Nacional de Investigación en Olericultura, Chapingo. 64 pp.

SÁncheZ-GonZÁlez, A. 2008. Una visión actual de la diversidad y distribución de los pinos en México. Madera y Bosque 14(1): 107-120.

Stead, J.W. 1983. A study of variation and taxonomy of the Pinus pseudostrobus complex. Commonwealth Forestry Review 62(1): 25-35.

TIASA. 1993. Programa de manejo para el aprovechamiento forestal maderable (1994-2004) de la comunidad de Ixtlán de Juárez, Oaxaca, México. Ixtlán, Oaxaca, México. 230 pp.

Toledo, V.M. 1989. La diversidad biológica de México. Ciencia y Desarrollo 14(81): 17-30.

Velázquez, A., J.F. Mas, R. Mayorga-Saucedo, J.R. Díaz, C. Alcántara, R. Castro, T. Fernández, J.L. Palacio, G. Bocco, G. Gómez-Rodríguez, L. Luna-GonzÁlez, I. Trejo, J. López-García, M. Palma, A. Peralta, J. Prado-Molina \& F. GonZÁlez-Medrano. 2002. Estado actual y dinámica de los recursos forestales de México. CONABIOBiodiversitas 41: 8-15.

Villar, R., J. Ruiz-Robleto, J.L. Quero, H. Poorter, F. Valladares \& T. Marañon. 2004. Tasas de crecimiento en especies leñosas: aspectos funcionales e implicaciones ecológicas. En: F. Valladares (ed.). Ecología del Bosque Mediterráneo en un Mundo Cambiante, pp. 191-227. Ministerio de Medio Ambiente, EGRAF, S.A. Madrid, España.

Viveros-Viveros, H., C. SÁenz-Romero, C.J. López-Upton \& J.J. VARGAS-HERnÁNDEZ. 2005a. Variación genética altitudinal en el crecimiento de plantas de Pinus pseudostrobus Lindl. en campo. Agrociencia 39: 575-587.

Viveros-Viveros, H., C. SÁenz-Romero \& R.R. Guzmán-Reyna. 2005b. Control genético de características de crecimiento en vivero de plántulas de Pinus oocarpa. Revista Fitotecnia Mexicana 28: 333-338.

Viveros-Viveros, H., C. SÁenz-Romero, J.J. Vargas-Hernández \& J. LópeZ-Upton. 2006. Variación entre procedencias de Pinus pseudostrobus establecidas en dos sitios de Michoacán, México. Revista Fitotecnia Mexicana 29: 121-126.

Zacarías-Eslava, Y., \& R. F. DEl Castillo. 2010. Comunidades vegetales templadas de la Sierra Juárez, Oaxaca: pisos altitudinales y sus posibles implicaciones ante el cambio climático. Boletín de la Sociedad Botánica Mexicana 87: 13-28.

Recibido: 05.01.15

Aceptado: 08.05.16 